\title{
Internals from Elaboration
}

\section{Discourse relations}

Elaboration or Narration, as so-called discourse relations (or rhetorical relations), are modelled in Segmented Discourse Structure Theory (SDRT) as relations between discourse constituents (or constituents for short). These are either propositions that come into being by interpretation of sentences occurring in a text; the propositions then have the status of DRSes. Or, constituents are compounds of such DRSes, constructed from DRSes (or compounds of them) by discourse relations. Elaboration and Narration in that sense, rather than referring to text types, provide links between constituents that allow them to combine in ways that, for a recipient, a resulting text is coherent and has (some) elaborative or narrative properties.

Interestingly, in SDRT the fact that two constituents $\alpha$ and $\beta$ are related by Elaboration is characterized partly in terms of other discourse relations. Notably there is an interaction with Narration and the Discourse Topic relation. For two constituents $\alpha$ and $\beta$, the claim is that Narration $(\alpha, \beta)$ is true, if and only if there is a third (implicit) constituent $\gamma$ that is elaborated by both $\alpha$ and $\beta$. As being elaborated by a constituent involves dominating that constituent, which in turn is a sufficient reason for making up that constituents discourse topic, $\gamma$ acts as their common discourse topic. The composite

claim can be articulated by means of the two SDRT axioms in (1) (in (1.b), $\delta \Downarrow \alpha$ says that $\delta$ dominates $\alpha$ ):

(1) a. Narration $(\alpha, \beta)$ iff $\exists \gamma[$ Elaboration $(\gamma, \alpha)$ \& Elaboration $(\gamma, \beta)]$

b. Elaboration $(\delta, \alpha) \rightarrow \delta \Downarrow \alpha$

\section{Two examples for Elaboration}

To appreciate the effect of the axioms one may look at a case where Elaboration obtains between explicit constituents, such as the text in (2).

(2) (K) Es regncte.

It rained

'It was raining.'

(E) Es fielen ungewöhnlich dicke Tropfen.

It fell unusually thick drops

'Unusually thick drops were falling down.' 
Here, the interpretation of $(\mathrm{K})(=\kappa)$ is elaborated by the interpretation of $(E)(=\varepsilon)$; according to (1.b), $\kappa \Downarrow \varepsilon$ obtains, which implies that $(\mathrm{K})$ expresses (E)'s discourse topic. However, the effect of (1.a) is that (the discourse topic) $\gamma$ dominates both $\alpha$ and $\beta$, and therefore is 'higher' than either of them in the hierarchy established by $\Downarrow$. Cf. the tree in (3):

(3)

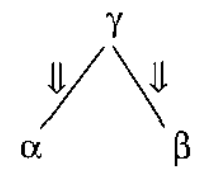

Texts that, for a given sentence (E), make explicit (E)'s discourse topic are used in Grabski (2000) to discuss the interaction of the discourse topic with sentence internally defined properties of $(\mathrm{E})$, i.e. its sentence topic, if it occurs ' ; (2.E) happens to have no sentence topic. A case of Elaboration where an (E) contains a sentence topic is (4.EI); the sentence topic is the NP enclosed in '[. $]_{\mathrm{T}}$ '. In this text $(\mathrm{K})$ is elaborated by both (E1) and (E2).

(4) (K) Nach dem 6. Schuljahr suchen die Freunde von Meryem verschiedene Schulen auf. After the 6. schoolyear attend the friends of $M$. different schools PART 'After the $6^{\text {th }}$ school year, Meyrem's friends will attend different schools.'

(E1) So wird [Hamdi] $\mathrm{T}$ glücklicherweise auf das nahe Gymnasium gehen. Thus will H. fortunately PREP the nearby Gymnasium go 'Thus, Hamadi will fortunately go to the nearby grammar school.'

(E2) Dagegen geht [Arkan] $]_{\mathrm{T}}$ vermutlich auf die Realschulc. However goes A. presumably PREP the secondary school 'Arkan however will presumably attend the secondary school.'

Elaboration is explicated in Grabski (2000) by means of an inferred object called a dimension. A dimension is a situation theoretic $(1+n$-ary) type that picks up an object $\mathrm{u}$ from the interpretation of $(\mathrm{K})$, the Elaborations 'frame', and distinguishes $\mathrm{n}$ further objects that are conceived of as having contrasting properties. Although the properties are introduced by the elaborating sentences (E-sentences) and make up new information in the text, they add information to the frame, that remains constant, and therefore no Continuation or Narration takes place; the text 'sticks to the discourse topic', so to speak. In (4), the frame is the referent of the NP die Freunde von Meryem, and there are 2 further objects (Hamdi and Arkan) that get contrasting properties by (the comment part of) the E-sentences.

\section{Dimensions}

Dimensions in the intended sense can be schematically notated as in (5.a), i.e. as objects in Extended Kamp Notation (EKN, see Barwise/Cooper 1993). These objects resemble notation used in DRT and in fact can be semantically related to it (see Cooper (1993)). (5.a) is a type that is obtained by abstracting out of the proposition represented in (5.b).

1 Occurence of sentence topics in German is here restricted to the syntactic position that has been considered in Frey (2000), i.e. in front of a sentence adverbial in the ,middle field' of a German sentence. 
(5) a.

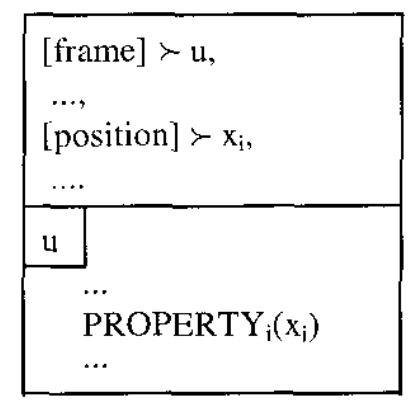

b.

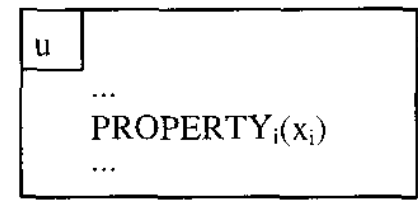

(5.b) says that the object $u$ is classified by PROPERTY ${ }_{i}\left(x_{i}\right)$ etc. (5.a) abstracts from this proposition by specifying two things: 1 . a set of parameters $\left(\mathrm{u}, \mathrm{x}_{\mathrm{i}}, \ldots\right)$ that are to be the arguments of the type and 2. a set of role indices (position ${ }_{\mathrm{i}}$ etc.). The latter act as 'labels' in the sense of Cooper (1992) and Glasbey (1994), i.e. as 'pieces of situated content' that restrict application of the argument role. The pieces of content may very well be anchored conceptually. To give an example, Cooper (1992) uses labels to model the cognitive distinction that is involved in the 'morning star paradox'. Although in (6.a and b) the same proposition is the result of applying a type to an assignment that has the object v(enus) as a concrete argument for the two occurring argument roles, only the assignment in (6.a) presents a (well-discussed) cognitive achievement:

(6) a.

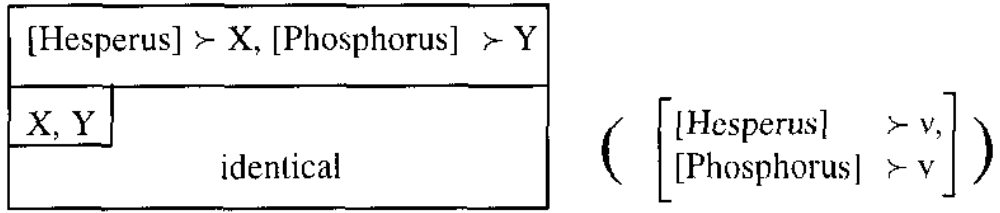

b.

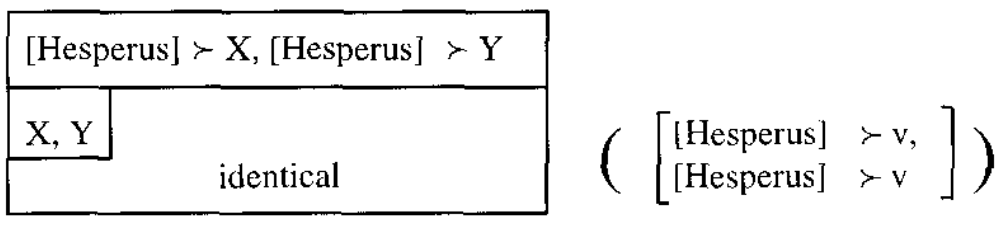

What is respected here is the truism that Hesperus and Phosphorus are cognitively related to two rather distinct situations; therefore their identification across these situations is the achievement it is. Cooper (1991) and Glasbey (1994) use role indices as labels also with respect to 'situated linguistic information', such as grammatical relations. Thus 'subject' or 'indirect object' are treated as ways in which respective referents are 'given'.

In our case, the 'frame' and 'position' roles of dimensions can be seen as labels in this sense, i.e. as information that is part of some assignment. Why should that be so? To appreciate this idea, one should start from the task of processing the second sentence of a text like (2): 
(2) (K) Es regnete.

It rained

'It was raining.'

(E) Es fielen ungewöhnlich dicke Tropfen.

It fell unusually thick drops

'Unusually thick drops were falling down.'

Processing (2.E) involves, according to SDRT, specifying a discourse relation that would attach (2.E)'s interpretation to the preceding text. In the simplest, default case this relation would be Narration. ${ }^{2}$ Is there a motivation to expect more? Intuitively this is the case, as (E) mentions that thick drops are falling down, which can be understood as elaborating the content expressed in $(\mathrm{K})$. But this intuition is world knowledge based. Nothing in the semantic structures of $(\mathrm{K})$ or $(\mathrm{E})$ in isolation can be used to infer this. On the other hand, with a different preceding sentence, $(\mathrm{E})$ may be interpreted as conveying a different discourse relation (Specification).

(7) (a) Gegen Nachmittag war es dann so weit.

PREP afternoon was it then so far

'In the afternoon things had developed so far.'

(E) Es fielen ungewöhnlich dicke Tropfen.

It fell unusually thick drops

'Unusually thick drops were falling down.'

The specific interaction of (E) with a preceding sentence, as well as the lack of a trigger for that interaction in (E) itself, gives these inferences of a discourse relation the status of bridging (cf. Asher/Lascarides (1998)), i.e. the inference of implicit additional information that is necessary for updating. Dimensions can be seen as being part of this implicit information. For example, whereas in (2), (E) has a preceding sentence that yields a frame in the intended sense, (E) in (7) has not.

Another observation concerns an interaction between E-sentences. (8) below is as (4), but with a modified second E-sentence (8.E2), that exhibits a different contrast than (4.E2). Whereas in (4), a contrast was established between school types, in (8) a contrast is established between close and distant schools.

(8) (K) Nach dem 6. Schuljahr suchen die Freunde von Meryem verschicdene Schulen auf. After the 6. schoolyear attend the friends of $M$, different schools PART 'After the $6^{\text {th }}$ school year, Meyrem's friends will attend different schools.'

(E1) So wird [Hamdi $]_{T}$ glücklicherweise auf das nahe Gymnasium gehen. Thus will H. fortunately PREP the nearby Gymnasium go 'Thus, Hamadi will fortunately go to the nearby grammar school.'

(E2) Dagegen geht [Arkan] ${ }_{\mathrm{T}}$ vermutlich auf eine Schule in einem anderen Bezirk. However goes A. presumably PREP a school PREP another district 'Arkan, however, will presumably attend a school in another district.'

To account for this difference, two distinct dimensions would have to be constructed. In both cases, their difference emerges only with the processing of the second E-sentence.

2 For the default role of Narration, cf. e.g. Lascarides et al. (1996) § 3.2. 
Construction of dimensions will be sketched further below. When doing this construction for a possible E-sentence $(E)$, we can conceive of the descriptive information that is given in specific $\mathrm{K}$ - and $\mathrm{E}$-sentences around, as situated information that partly determines the label under which $(\mathrm{E})$ is contrastive within the dimension at hand. Thus it is the fact that in $(2 . \mathrm{K})$ the intended situation is described as being rainy that allows to interprete descriptive information articulated in (2.E) as expressing 'a way in which it rained' in that situation. Analogously, descriptive information works when (4.E2) and (8.E2) are processed. That is, if the possibility of Elaboration is present, construction of a dimension will start with a proposition that states that the (putative) frame is classified by the content of the (putative) E-sentence. The result is a proposition as schematically given in (5.b). What then has to be done is to 'abstract out' of this proposition, thereby constructing labels that impose contrastive roles $^{3}$ on the objects classified by the dimension. The latter is established as a type, as it is schematically given in (5.a).

The construction proposed in the following section makes use of another, interfering idea, that concerns the relation between what is said in the $\mathrm{K}$ - and E-sentences. In Barwise (1989) the effect of projecting a given n-ary relation on a relation with $n-1$ arguments is discussed. The result is a loss of information with respect to the dimension of variation that otherwise is 'kept open' by the cancelled argument role. An example used by Barwise is the relation between ( 9 a and $b$ ).

(9) a. It's 4 o'clock.

b. It's 4 o'clock here.

Whereas (9.b) refers to a time zone, (9.a) does not. Reference to time zones is necessary only in certain situations. If there is no communication over times zones, they are not discriminated. The idea is that in a $\mathrm{K}$-sentence that is to be elaborated, there is a similar absence of distinctions introduced first by the E-sentences. Or, an E-sentence expresses the same proposition as its $\mathrm{K}$-sentence, but enriches the internal classifying type involved by an additional argument role, a role that was not there before. (The dimension then acts as a restriction on that additional argument.)

3 That the labels are the place where to settle the contrastive information is supported by Elaboration texts where contrastiveness is not ensured by sentence comments, as in

(i) (K) Die Gestirne haben alle die gleiche Gestalt.

The stars have all the same shape

'The stars have all the same shape.'

(E1) Der Mond ist rund.

The moon is round

'The moon is round.'

(E2) Die Erde ist ebenfalls rund.

The earth is as well round

'The earth is round as well.'

(E3) Auch die Sonne ist rund.

Also the sun is round

'Also the sun is round.' 


\section{Dimension constructions}

(2) illustrates that Elaboration may obtain without sentence topics occurring in the actual E-sentence. As worked out in Grabski (2000), analysis of Elaboration in a case like (2) can start with seeing them as having roles in two 'Austinian' propositions (cf. $\operatorname{Kim}(1998)$ ). Call the respective situations $s_{\mathrm{k}}$ and $s_{\mathrm{e}}$, the propositions $\kappa$ and $\varepsilon$. That Elaboration obtains between $\kappa$ and $\varepsilon$ is based on the possibility to construct a complex enriched classification for a token that is present in $\kappa$ and $\varepsilon$. Relations between classifications have been studied extensively in Channel Theory. Using the notion of a channel theoretic constraint (cf. Cavedon (1995), Cavedon/Glasbey (1996)), we may say that $s_{\mathbf{k}}$ and $s_{e}$ are of a type ELABORATES, if they also are of some complex type $\phi$ that encodes the structural preconditions for Elaboration.

In channel theoretic terms, we then have a constraint

(10) $\phi \rightarrow$ ELABORATES

that classifies two connections (tokens that have the shape of pairs of objects) ' $S_{\mathfrak{e}} \mapsto S_{\mathfrak{e}}{ }^{\prime}$ and ' $s_{k} \mapsto s_{k}{ }^{\prime}$. In that case, $s_{e}$ and $s_{k}$ have to be of the types that form the antecedent and the succedent of the constraint. They act as 'signals': given their being of type $\phi$, and given the constraint, it can be inferred that they are of type ELABORATES.

Initial steps in the construction of $\phi$ for an analysis of (2) are $\kappa$ and $\varepsilon$ themselves, represented in $(11 . \mathrm{a}, \mathrm{b})$. (Interpretation of tenses etc. is suppressed.)

(11) a.

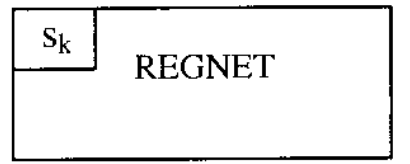

b.

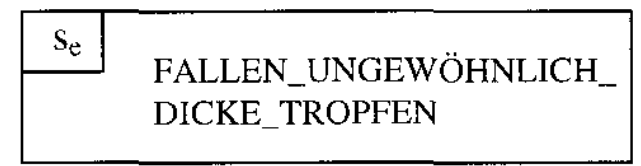

A suitable dimension is a type $\delta$ such that

- the internal type $\tau$ of $\varepsilon$, predicated of an additional argument $x_{1}$, is an internal type $\tau^{\prime}$ in $\delta$,

- the proposition $\mathrm{p}$, that the (frame object) $\mathrm{u}$ is classified as the expanded type $\tau$, is stated,

- $\delta$ is constructed by abstracting out of $\mathrm{p}$ with appropriate argument roles, that bind $\mathrm{u}$ and $x_{i}$. 'Appropriate' means that role indices are chosen appropriately; that is, $u$ is bound by [frame], and $x_{i}$ is bound by a role index that 1 . secures descriptive contrastiveness with other possible arguments and 2. (loosely:) is restricted by a descriptive relation between the internal types of $\kappa$ and $\varepsilon$.

These clauses result, in the case of (2), in a dimension as $\delta$ in (12), that, in its turn can be applied to a frame $s_{k}$ and a manner entity $m$, to get the status of a proposition:

\footnotetext{
4 This account seems more correct than the constraint given in Grabski (2000), section 7.2.
} 
(12)

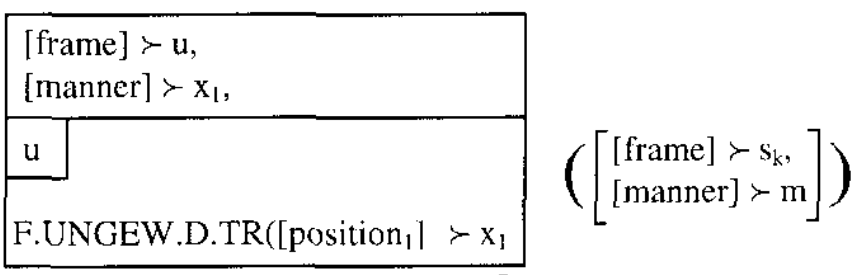

$\{\delta\}$

The construction of $\phi$ now uses the idea of 'reversed projection', taking into account the setting of the processing of the E-sentence. In this setting, the interpreter has to start from the actual constituent and its ingredients, in this case $\varepsilon$ and the one token obtainable, $s_{\mathrm{e}}$. If $s_{\mathrm{e}}$ can be classified just as $s_{\mathrm{k}}$ is classified in $\kappa$, with a certain modification, then accepting ELABORATES is warranted. The modification is that the internal type that does $\mathrm{s}_{\mathrm{e}}$ 's classification has an additional argument role, that in turn is restricted by the background dimension.

A representation for this (i.e. the type $\phi$ ) is in (13). Included in the restriction box (the box to the right of the double bar), the proposition in (12) behaves like a presupposition on the assertion made within (13) (contained in the box to the left of the double bar; cf. Barwise/ Cooper 1993). That proposition concerns the properties of $s_{e}$ in the presence of the preceding $\kappa$. The internal type REGNET has been enriched with an additional argument role, the components of which are restricted by the dimension and the assigment to it, in that the role indices in the dimension's abstraction fix u's role as that of a 'frame' and $x_{1}$ 's role as that of 'manner'. The manner m's property is fixed descriptively by $\delta$ 's internal type'.

$\phi$ not only plays a role in the constraint in (10). It is also the succedent type in another constraint ' $\varepsilon \rightarrow \phi$ ', that has to be used before (10) is used. Actually, different versions of $\phi$ have to be provided, to account for varieties of processing situations. ${ }^{6}$ Thus, a slightly different situation arises when a second E-sentence, e.g. (E2) in (4), is processed; then a second position on some dimension is inferred.

(13)

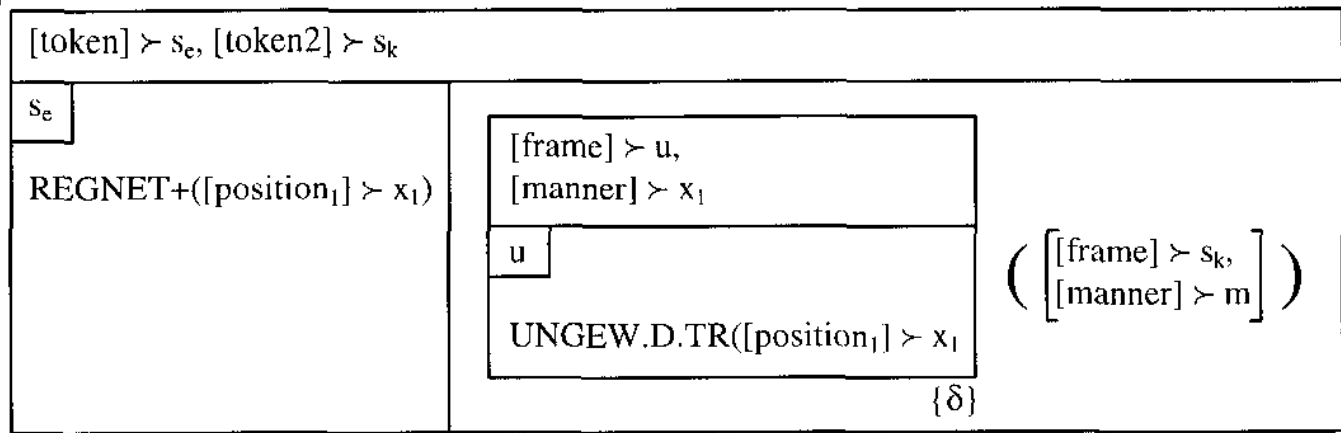

Finally, the E-sentences in (4) contain sentence topics. Their role in an Elaboration context such as (4) is interpreted in Grabski (2000) as providing the arguments of the dimension. The referent a of some sentence topic establishes an additional connection ' $\mathrm{a} \mapsto \mathrm{a}$ ' that is classified by an appropriate version of ' $\varepsilon \rightarrow \phi$ '. The interaction between sentence and discourse topics would then turn out to consist in a fixing of the latter by the former, a fixing that is mediated by some dimension.

5 The treatment of 'manner' here is only a provisional solution.

6 Cf. Grabski (2000) 


\title{
References
}

Asher, N. (1993): Reference to Abstract Objects in Discourse. Dordrecht

Asher, N.; Lascarides, A. (1998): Bridging, Journal of Semantics 15.1. 83-113

Barwise, J. (1989): Situations, Facts, and True Propositions. In: Barwise, J.: The Situation in Logic. Stanford. (=CSLI Lecture Notes 17) 221-254

Barwise, J.; Cooper R. (1993): Extended Kamp Notation: A Graphical Notation for Situation Theory. In: Aczel, P. et al. (eds.): Situation Theory and its Applications. Vol. 3. Stanford. (=CSLI Lecture Notes 37) $29-54$

Cavedon, L. (1995): A Channel Theoretic Approach to Conditional Reasoning. PhD. Dissertation. Edinburgh University.

Cavedon, L.; Glasbey, S. (1994): Outline of an Information-Flow Model of Generics. Acta Linguistica Hungarica $42.227-245$

Cooper, R. (1991): Three Lectures on Situation Theoretic Grammar. In: Filgueiras, M. ct al. (eds.): Natural Language Proceedings. EALA 90, Proceedings. Berlin; New York. (=Lecture Notes in Artificial Intelligence 476). 101-104

Cooper, R. (1992): A working person's guide to situation theory. To appear in: Hansen, S. L.; Sörensen, F. (eds.): Topics in Semantic Representation. Frederiksberg. Available at: http://www.ling.gu.se/ cooper/papers.html

Cooper, R. (1993): Towards a general semantic framework. In: Cooper, R. (cd.): Integrating Semantic Theories. Dyana-2. Esprit Basic Research Project 6852. Deliverable R2.1.A. 51-97

Frey, W. (2000): Über die syntaktische Position des Satztopiks im Deutschen. In: Schwabe et al. (eds.). $137-172$

Frey, W. (2001): A Medial Topic Position for German. Ms. Zas, Bcrlin

Glasbey, S. (1994): Event Structure in Natural Language Discourse. PhD. Thesis. Edinburgh University

Grabski, M. (2000): Satztopik und Diskurstopik in Elaboration-Kontexten. In: Schwabe et al. (eds.). 173207

Kim, Y. (1998): Information Articulation and Truth Conditions of Existential Sentences. Language and Information 1.1

Lascarides, A.; Copestake, A.; Briscoe, T. (1996): Ambiguity and Coherence. Ms. Edinburgh

Schwabe, K. et al. (eds.): Issues on Topics. (=ZAS Papers in Linguistics 20)

\author{
Michacl Grabski \\ Zentrum für allgemeine Sprachwissenschaft, \\ Typologie und Universalienforschung \\ Jägerstr. 10-11 \\ 10117 Berlin \\ Germany \\ grabski@zas.gwz-berlin.de
}

